\title{
Essais de conditionnement morpholine du circuit eau-vapeur de la centralle de Fessenheim
}

\section{Test of morpholine treatment of the water-steam circuit in the Fessenheim power station}

\author{
J. Grégoire \\ EDF, GRPT-Est, Strasbourg
}

\section{Généralités}

Les deux tranches de la centrale nucléaire de Fessenheim constituent la tête de série des tranches 900 MWe PWR du programme nucléaire français. Elles ont été respectivement mises en service en avril et octobre 1977.

\section{Description sommaire de l'installation (voir Fig. 1)}

Chaque tranche comporte une chaudière nucléaire et un groupe turbo-alternateur.

La chaudière est constituée du réacteur nucléaire proprement dit, fournissant l'énergie thermique, des pompes primaires véhiculant le fluide caloporteur (eau sous pression) et de trois générateurs de vapeur (GV) formés de tubes en U. Le fluide caloporteur circule à l'intérieur de ces tubes (appelés aussi côté "primaire"). A l'extérieur des tubes (côté "secondaire"), l'eau alimentaire des GV, préchauffée dans le poste d'eau, s'échauffe dans le faisceau tubulaire et se vaporise au-dessus de celui-ci. La vapeur ainsi produite traverse des séparateurs d'humidité intégrés aux générateurs de vapeur d'où elle sort à 57 bars pour alimenter la turbine.

La turbine proprement dite comporte un corps HP et trois corps BP. La vapeur est détendue jusqu'à 9 bars dans le corps HP où son titre passe de $0,25 \%$ (admission) à environ $10 \%$ (échappement). Cette vapeur humide est séchée et surchauffée dans les sécheurssurchauffeurs dont les condensats sont renvoyés dans le poste d'eau; les caractéristiques thermodynamiques de la vapeur sont ainsi considérablement améliorées à l'admission des corps BP. Après détente dans les corps $B P$, la vapeur se condense dans le condenseur refroidi par l'eau du Grand Canal d'Alsace. Cette eau est reprise par des pompes d'extraction, réchauffée dans le poste d'eau BP, et à l'aide des pompes alimentaires réinjectée dans les GV à travers le poste de réchauffage HP. L'ensemble générateur de vapeur (côté secondaire), tuyauteries vapeur, turbine, sécheurs-surchauffeurs, condenseur et poste d'eau, constitue le circuit eauvapeur (ou circuit secondaire).

L'objet de cet article porte sur les essais de conditionnement chimique du "circuit secondaire" effectués par la Section Chimie du Groupe Régional de Production Thermique EST.

\section{Nature des matériaux}

Générateur de vapeur Inconel : $72 \% \mathrm{Ni}, 14$ à $17 \% \mathrm{Cr}, 6$ à $7 \% \mathrm{Fe}$

Tuyauteries vapeur, sécheurs-surchauffeurs, turbine

Acier au carbone A 48 $(0,22 \%)$ (sauf les parties mobiles de la turbine)

Poste de réchauffage BP (R1 R2 R3)

Poste de réchauffage

HP (R4 R5 R6)

Condenseur

Laiton $(70 \% \mathrm{Cu}, 30 \% \mathrm{Zn})$

Acier au carbone

Laiton $(70 \% \mathrm{Cu}, 30 \% \mathrm{Zn})$

\section{Conditionnement chimique du circuit eau-vapeur}

Il convient de rappeler que le conditionnement préconisé par Westinghouse a subi plusieurs modifications suite aux difficultés rencontrées sur certaines unités PWR. Nous donnons ci-dessous en bref l'historique de son évolution.

Le choix du matériau des tubes GV s'est porté sur l'Inconel 600 moins sensible à la corrosion sous tension provoquée par les chlorures que l'acier inox. On s'est aperçu à l'usage que cet alliage était très sensible à la corrosion fissurante sous tension particulièrement en milieu alcalin, d'où le passage du conditionnement 


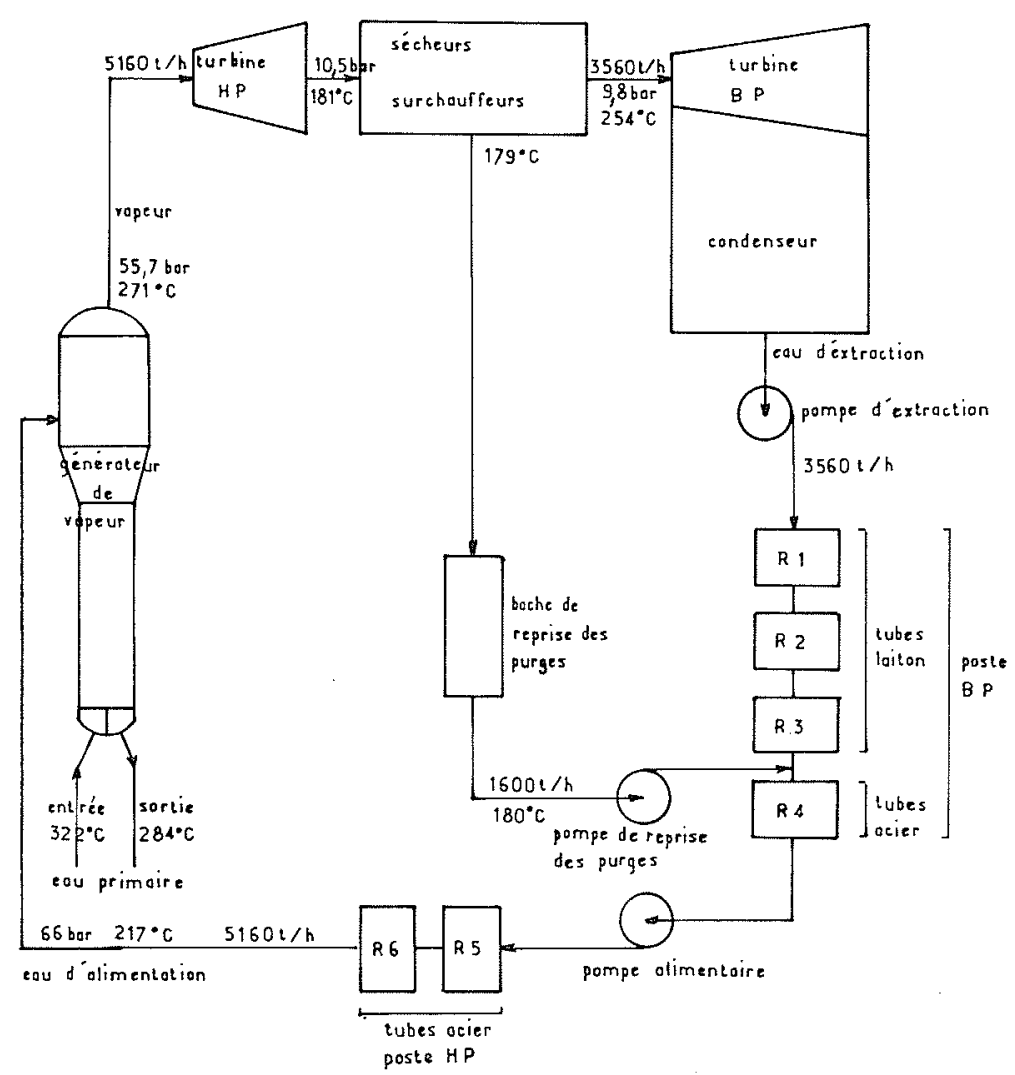

Figure 1 - Schéma simplifié du circuit secondaire de Fessenheim.

volatil avec injection occasionnelle de $\mathrm{PO}_{4} \mathrm{Na}_{3}$ en cas d'entrée d'eau brute à un conditionnement phosphate pur avec:

$$
2,2<\frac{\mathrm{Na}}{\mathrm{PO}_{4}}<2,6
$$

Le respect de cette fourchette devait éviter la formation de soude libre. Ce conditionnement a dû également être rapidement abandonné (1975) ; le phosphate a en effet provoqué en se séquestrant sur les tubes une attaque acide et un amincissement généralisé de ces tubes. A la même époque on a constaté également une striction des tubes en Inconel par croissance de l'oxyde au niveau des interstices entre plaques intermédiaires et tubes (connus sous le nom de phénomène de "denting").

Ces deux phénomènes sont à l'origine de la reprise du conditionnement volatil, sans le secours du phosphate en cas d'entrée d'eau brute. C'est ce dernier type de conditionnement qui a été retenu dès le démarrage de la centrale de Fessenheim. Un tel conditionnement impose le maintien d'une salinité dans les GV relativement faible $\left(\lambda^{+} \mathrm{GV}(1)<2 \mu \mathrm{S} / \mathrm{cm}\right)^{(1)}$; en effet toute entrée d'eau brute peut provoquer une attaque acide, dans le cas d'eau de mer et d'estuaires (présence de $\mathrm{Cl}_{2} \mathrm{Mg}$ à hydrolyse acide) mais également dans le cas d'eaux de certaines rivières ( $\mathrm{TACi}>\mathrm{TAC}$ ). Dans le cas d'eaux où le $\mathrm{TACi}<\mathrm{TAC}$, les chlorures par eux-mêmes sont également source de corrosion en présence d'oxygène.

$\mathrm{La}$ concentration en ions $\mathrm{OH}$ libres dans les GV

(1) $\lambda^{+} \mathrm{GV}$ : conductivité cationique de l'eau des générateurs de vapeur. doit également rester limitée. La corrélation normale que l'on vérifie pour une eau donnée entre le Na présent et les conductivités cationiques, permet de connaître la soude libre apportée dans les GV par l'eau d'appoint ou par les entrées d'eau brute, (voir Fig. 2).

Compte tenu des problèmes, liés à la chimie, rencontrés sur un certain nombre de centrales de ce type, l'exploitant de Fessenheim a attaché une attention particulière au conditionnement chimique dès le démarrage de la centrale.

Cependant, la présence de quantités importantes de matières en suspension dans le circuit eau-vapeur et l'examen visuel des tuyauteries de liaison du corps HP aux sécheurs-surchauffeurs, ont mis en évidence une forme de corrosion déjà connue par ailleurs, à savoir le phénomène d'érosion-corrosion des circuits en vapeur humide.

Sur recommandations d'Alsthom, une première mesure a été prise qui consistait à relever le pH de 9 à 9,3 en conditionnement ammoniaque. Cette mesure s'avérant insuffisante, le remplacement de l'ammoniaque par de la morpholine a peu être envisagé.

Intérêt théorique d'un conditionnement morpholine à $\mathrm{pH}$ de 9,3

\section{Mécanisme de la formation de la couche de magnétite des aciers au carbone}

La formation de la magnétite, en milieu aqueux, se fait par le processus de la double couche (théorie de Potter et Mann). 


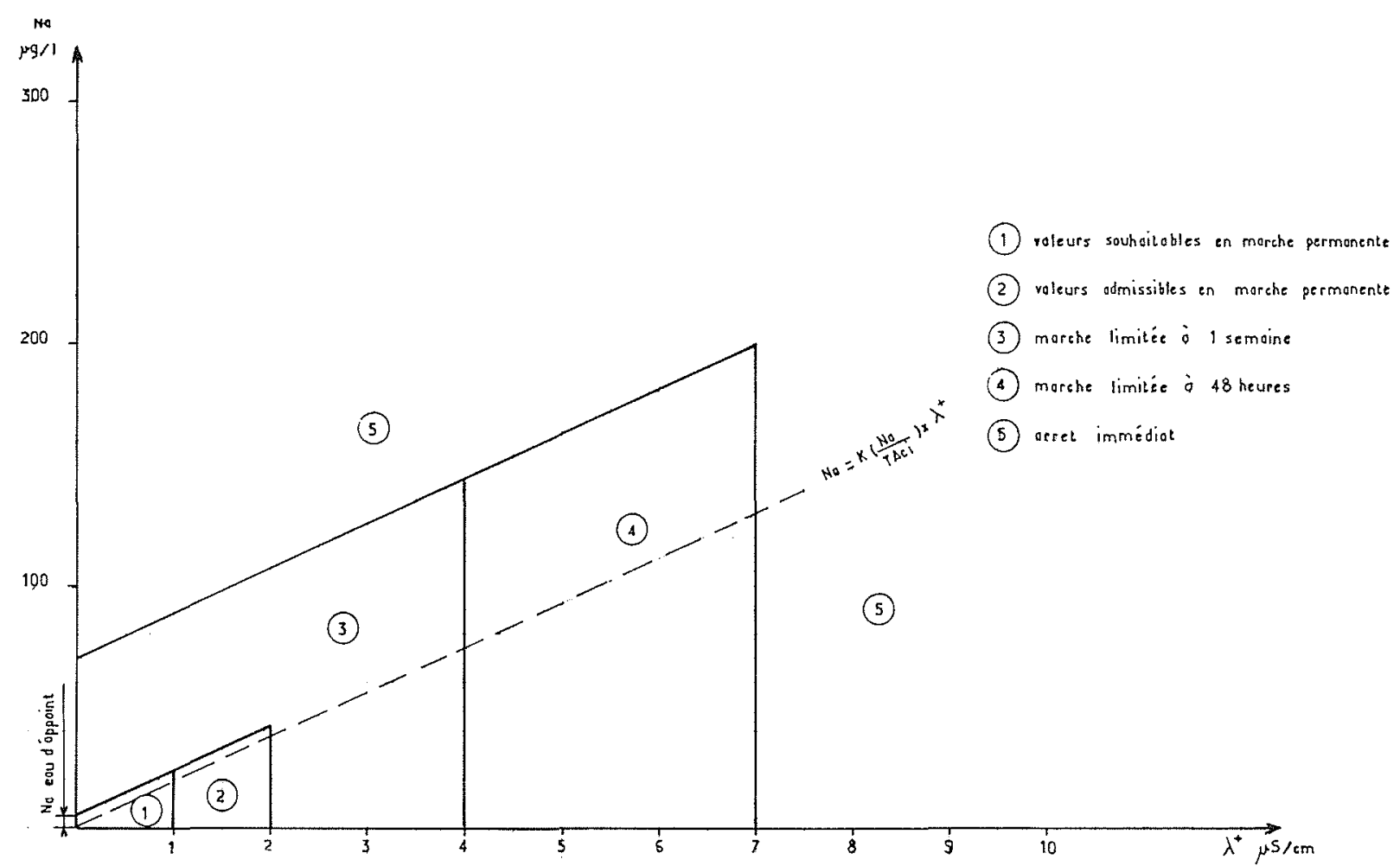

Figure 2 - Consignes d'exploitation.

1) Développement d'une couche interne de magnétite par action directe de l'eau (agent oxydant) sur la surface métallique. La porosité de cette couche est d'après Homig d'environ $15 \%$.

2) Formation d'une couche externe protectrice par migration des ions $\mathrm{Fe}^{++}$à travers la couche interne, formation de $\mathrm{Fe}(\mathrm{OH})_{2}$ au voisinage de la paroi et transformation en magnétite selon la réaction :

$$
3 \mathrm{Fe}(\mathrm{OH})_{2} \rightarrow \mathrm{Fe}_{3} \mathrm{O}_{4}+2 \mathrm{H}_{2} \mathrm{O}+\mathrm{H} 2
$$

(réaction de Schikorr)

Si la vitesse du fluide est trop élevée, la concentration en $\mathrm{Fe}(\mathrm{OH})_{2}$ au vosinage des parois est insuffisante et la couche externe protectrice se forme peu ou pas: c'est le phénomène d'érosion-corrosion. Toutes choses égales par ailleurs, ce phénomène st d'autant moins marqué que :

- la température est plus élevée (augmentation de la vitesse de la réaction de Schikorr);

- le pH est plus élevé (augmentation de la vitesse de formation de $\left.\mathrm{Fe}(\mathrm{OH})_{2}\right)$.

Ainsi donc, pour une température donnée, un accroissement de $\mathrm{pH}$ doit se traduire par une diminution des phénomènes d'érosion-corrosion.

\section{Comparaison des $\mathrm{pH}$ obtenus dans le film liquide contre les parois entre les conditionnements ammoniaque et morpholine}

Pour les coefficients de partage (concentration en phase vapeur/concentration en phase liquide) de l'ammoniaque $(3,7)$ et de la morpholine $(1,0)$ et une humi- dité moyenne de $10 \%$, on obtient des $\mathrm{pH}$ de film liquide à $250^{\circ} \mathrm{C}$, fonction du mode de conditionnement et du pH vapeur.

\begin{tabular}{|c|c|c|}
\hline \multirow{2}{*}{$\begin{array}{c}\text { pH vapeur } \\
\text { à } 25^{\circ} \mathrm{C}\end{array}$} & \multicolumn{2}{|c|}{$\mathrm{PH}$ du film liquide à $250^{\circ} \mathrm{C}$} \\
\cline { 2 - 3 } & $\begin{array}{c}\text { Conditionnement } \\
\text { ammoniaque }\end{array}$ & $\begin{array}{c}\text { Conditionnement } \\
\text { morpholine }\end{array}$ \\
\hline 9 & 5,76 & 6,10 \\
9,1 & 5,80 & 6,20 \\
9,2 & 5,85 & 6,30 \\
9,3 & 5,90 & 6,41 \\
9,4 & 5,95 & 6,52 \\
\hline
\end{tabular}

Rappelons que le $\mathrm{pH}$ de neutralité de l'eau à $250^{\circ} \mathrm{C}$ est de 5,62 .

Il apparait donc, compte tenu du coefficient de partage défavorable de l'ammoniaque par rapport à la morpholine et de sa basicité à $250^{\circ} \mathrm{C}$ pratiquement équivalente à celle de la morpholine, qu'un conditionnement ammoniaque à un $\mathrm{pH}$ de 9,4 dans la vapeur (irréalisable en raison des corrosions des alliages cuivreux) ne serait même pas équivalent à un conditionnement morpholine à $\mathrm{pH} 9$. Ceci démontre bien la supériorité de la morpholine pour augmenter le $\mathrm{pH}$ de la phase liquide.

\section{Essais de conditionnement morpholine à la centrale de Fessenheim}

Ces essais avaient pour but de comparer à la centrale de Fessenheim le comportement des circuits eau-vapeur de deux tranches, l'une étant conditionnée à l'ammo- 
niaque et l'autre à la morpholine avec le même $\mathrm{pH}(9,3)$ dans la vapeur. Un schéma simplifié du circuit secondaire est représenté sur la figure 1 .

Pour cette étude, les paramètres chimiques contrôlés ont été :

1) Les matières en suspension, mesurées par intégration sur filtre millipore (prélèvement de $12 \mathrm{~h}$, la nuit, en l'absence de variations de débits d'échantillonnage).

2) Les teneurs en hydrogène, mesurées par chromatographie en phase gazeuse (appareil Girdel).

Contrôles des matières en suspension (tableaux 1,2 et 3)

1) On constate avec les deux types de conditionnement que les teneurs de matières en suspension de l'eau alimentaire et de l'eau d'extraction sont très variables $(8$ à $44 \mu \mathrm{g} / \mathrm{kg}$ de fer total à l'alimentation, 3 à $13 \mu \mathrm{g} / \mathrm{kg}$ à l'extraction). L'ensemble des valeurs de l'alimentation est cependant plus élevé que celles de l'extraction, la différence pouvant donc concerner l'érosion-corrosion des circuits vapeur HP.

2) La comparaison des conditionnements morpholine et ammoniaque à pH de 9,3 dans la phase vapeur montrent que la corrosion généralisée du fer est diminuée d'un facteur 3 avec la morpholine (16 contre $47 \mu \mathrm{g} / 1$ dans l'eau alimentaire et $16 \mu \mathrm{g} / 1$ contre $57 \mu \mathrm{g} / 1$ dans les purges sécheurs). En réalité l'examen visuel de la turbine HP et des tuyauteries de liaison de Fessenheim montre que les attaques ont été atténuées d'un facteur nettement supérieur à 3 lors du passage en conditionnement morpholine. Ceci est. également confirmé par les

\begin{tabular}{|c|c|c|c|c|c|c|c|c|c|}
\hline \multicolumn{2}{|c|}{ Tableau 1 - Evolution des teneurs en cuivre et fer dans le circuit secondaire de la tranche 1} \\
(conditionnement morpholine)
\end{tabular}

\begin{tabular}{|c|c|c|}
\hline \multicolumn{3}{|c|}{$\begin{array}{c}\text { Tableau } 2 \text { - Evolution des teneurs de matières en } \\
\text { suspension sur les purges sécheurs } \\
(\mu \mathrm{g} / \mathrm{l}) \text { tranche } 1\end{array}$} \\
\hline Dates & $\begin{array}{c}\text { Matières en } \\
\text { suspension en } \mu \mathrm{g} / \mathrm{l}\end{array}$ & Remarques \\
\hline $\begin{array}{l}16.03 .1978 \\
17.03 .1978\end{array}$ & $\begin{array}{l}57 \\
55\end{array}$ & $\begin{array}{l}\text { Conditionnement } \\
\text { ammoniaque } \\
\text { Charge : } 920 \mathrm{MW}\end{array}$ \\
\hline $\begin{array}{l}27.04 .1978 \\
28.04 .1978\end{array}$ & $\begin{array}{l}19 \\
14\end{array}$ & $\begin{array}{c}\text { Conditionnement } \\
\text { morpholine depuis } \\
\text { le } 17.04 .1978 \\
\text { Charge : } 920 \mathrm{MW}\end{array}$ \\
\hline
\end{tabular}

constatations effectuées à la centrale belgo-française de Tihange où les pertes d'épaisseur pendant la période de fonctionnement en conditionnement morpholine sont pratiquement nulles.

3) Les différences constatées en ce qui concerne la corrosion généralisée du cuivre avec les 2 types de conditionnement ne sont pas assez significatives pour être prises en considération. Par contre, ponctuellement, au niveau des risques d'attaque des refroidisseurs d'air, le conditionnement morpholine apparaît préférable puisqu'il supprime les risques de corrosion ammoniacale du laiton des tubes ('ammoniaque peut en effet se concentrer au niveau des refroidisseurs d'air à des teneurs de plusieurs $\mathrm{mg} / \mathrm{kg}$ ). 


\begin{tabular}{|c|c|c|c|c|c|c|c|c|c|}
\hline \multicolumn{10}{|c|}{$\begin{array}{c}\text { Tableau } 3 \text { - Evolution des teneurs en cuivre et en fer dans le circuit secondaire de la tranche } 2 \\
\text { (conditionnement ammoniaque-hydrazine) }\end{array}$} \\
\hline \multirow{2}{*}{$\begin{array}{c}\text { Charge } \\
\text { (MW) }\end{array}$} & \multirow[b]{2}{*}{ Dates } & \multicolumn{3}{|c|}{ Extraction } & \multicolumn{5}{|c|}{ Alimentation } \\
\hline & & $\begin{array}{l}\text { Cu total } \\
\mu \mathrm{g} / \mathrm{l}\end{array}$ & $\begin{array}{c}\mathrm{O}_{2} \\
\mu \mathrm{g} / 1\end{array}$ & $\begin{array}{l}\text { Fe total } \\
\mu \mathrm{g} / 1\end{array}$ & $\begin{array}{l}\text { Cu total } \\
\mu \mathrm{g} / \mathrm{l}\end{array}$ & $\begin{array}{l}\text { Fe total } \\
\mu \mathrm{g} / \mathrm{l}\end{array}$ & $\begin{array}{ll}\mathrm{pH} \\
\text { à } 25^{\circ} \mathrm{C}\end{array}$ & $\begin{array}{l}\text { Morpholine } \\
\mathrm{mg} / \mathrm{l}\end{array}$ & $\underset{\mu \mathrm{g} / \mathrm{l}}{\text { Ammoniaque }}$ \\
\hline 900 & 04.10 .1978 & 2 & 9 & 13 & 2 & 36 & 9,25 & - & 700 \\
\hline 900 & 05.10 .1978 & 3 & 10 & 10 & 1 & 29 & 9,30 & - & 860 \\
\hline 900 & 06.10 .1978 & 1 & 10 & 7 & 1 & 38 & 9,20 & - & 560 \\
\hline 900 & 11.10 .1978 & 2 & 23 & 11 & 2 & 33 & 9,10 & - & 350 \\
\hline 870 & 25.01 .1979 & 4 & 10 & 49 & 2 & 38 & 9,10 & - & - \\
\hline 875 & 26.01.1979 & 3 & 10 & 18 & 2 & 35 & 9,35 & - & - \\
\hline 920 & 31.01 .1979 & 2 & 6 & 38 & 1 & 26 & 9,40 & - & 1035 \\
\hline 910 & 01.02 .1979 & 2 & 6 & 25 & 4 & 79 & 9,25 & - & - \\
\hline 910 & 02.02 .1979 & 2 & 6 & 19 & 2 & 55 & 9,20 & - & - \\
\hline 900 & 07.02 .1979 & 1 & 9 & 14 & 1 & 50 & 9,15 & - & 450 \\
\hline \multirow[t]{2}{*}{905} & 09.02 .1979 & 2 & 8 & 25 & 2 & 93 & 9,25 & - & - \\
\hline & Moyenne & 2 & & 21 & 2 & 46 & & & \\
\hline
\end{tabular}

L'examen de ces refroidisseurs d'air après la première année de fonctionnement a confirmé l'attaque ammoniacale du refroidisseur d'air de la tranche 2 conditionnée à l'ammoniaque. De plus on a constaté sur cette tranche la corrosion ammoniacale des rouets en bronze des pompes à vide.

4) Sur la tranche 1, le passage du conditionnement morpholine à un conditionnement ammoniaque a montré après $400 \mathrm{~h}$ de fonctionnement stable, une augmentation des matières en suspension d'un facteur $3(16 \mu \mathrm{g} / 1$ contre $46 \mu \mathrm{g} / 1)$. Cela signifie que la reprise du conditionnement ammoniaque dépassive assez rapidement les tuyauteries. Inversement, les basses valeurs de matières en suspension mesurées dès le début du conditionnement morpholine montre que la passivation des tuyauteries est également rapide.

\section{Contrôles des teneurs en $\mathrm{H}_{2}$ dans les circuits (Fig. 3)}

Les analyses d'hydrogène effectuées permettent de tirer les enseignements suivants :

1) La production d'hydrogène dans le circuit vapeur HP (différence entre la sortie sécheur-surchauffeur et la vapeur vive) apparaît en baisse progressive durant tout le conditionnement morpholine (passage de $1,5 \mu \mathrm{g} / 1$ en début de traitement à $0,1 \mu \mathrm{g} / 1$ en fin de traitement). Il est à remarquer que sur la tranche 2 , conditionnée à l'ammoniaque, la production d'hydrogène dans les circuits vapeur se trouve également voisine de $1,5 \mu \mathrm{g} / 1$ (valeur relevée sur la tranche 1 avant le conditionnement morpholine).

2) La production d'hydrogène dans le poste d'eau de la tranche 1 suit les fluctuations de la production d'hydrogène dans les circuits vapeur (retour $\mathrm{d}^{\prime} \mathrm{H}_{2}$ en provenance des soutirages 4-5-6).

En définitive, la réduction des teneurs en hydrogène (facteur supérieur à 10) corrobore très bien les obser- vations faites sur le matériel et la réduction très importante des attaques à l'échappement HP.

\section{Rôle du $\mathrm{CO}_{2}$ sur les deux types de conditionnement}

Concentration du $\mathrm{CO}_{2}$ dans les générateurs de vapeur

Des mesures de $\mathrm{CO}_{2}$ dans les $\mathrm{GV}$ effectuées avec les 2 conditionnements (voir tableau 4) montrent que les teneurs sont toujours plus élevées en conditionnement morpholine qu'en conditonnement ammoniaque. Pour en connaitre la cause, un bilan massique de $\mathrm{CO}_{2}$ a été effectué dans les circuits avec les 2 conditionnements. D'après ce bilan, il apparaît avec les 2 conditionnements, en marche normale et sans entrée d'air particulière, que les quantités de $\mathrm{CO}_{2}$ introduites, proviennent pour environ $80 \%$ du $\mathrm{CO}_{2}$ piégé à l'état ionisé, à partir des entrées d'air au condenseur, pour $15 \%$ des réactifs et pour $5 \%$ de l'eau d'appoint partiellement carbonatée.

Un piégeage du $\mathrm{CO}_{2}$ de l'eau d'appoint et des réactifs s'avère donc marginal pour diminuer les teneurs en $\mathrm{CO}_{2}$ à l'équilibre dans les GV. Ceci a pu être vérifié expérimentalement à la centrale de Fessenheim. Après mise au niveau haut du condenseur une coupure de l'appoint durant $10 \mathrm{~h}$ n'a provoqué aucune baisse significative de la conductivité cationique des GV. De même une coupure des réactifs durant le même temps n'a entraîné qu'une baisse de conductivité de l'ordre de $0,1 \mu \mathrm{S} / \mathrm{cm}$.

Par contre, on constate qu'une mauvaise extraction d'air au condenseur par suite de fonctionnement défectueux des pompes à vide, provoque des variations de conductivité cationique $\mathrm{GV}$ parfois supérieures à $0,3 \mu \mathrm{S} / \mathrm{cm}$.

Les quantités d'air introduites dans les circuits étant voisines avec les deux types de conditionnement, les différences de teneurs en $\mathrm{CO}_{2}$ à l'équilibre dans les $\mathrm{GV}$ peuvent donc provenir de la nature du réactif de conditionnement lui-même. Le $\mathrm{CO}_{2}$ a en effet plus d'affinité 
312

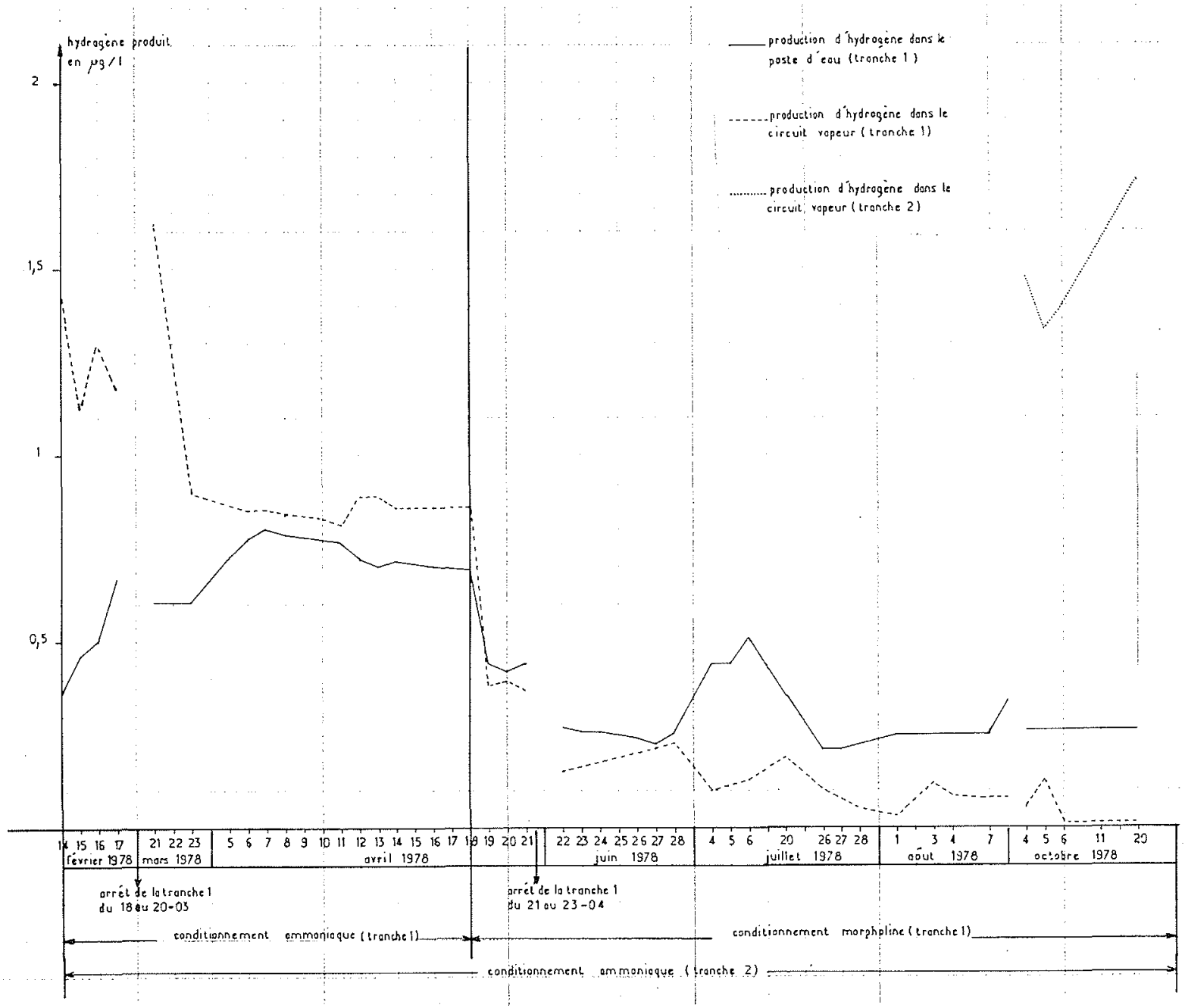

Figure 3 - Essais comparatifs de conditionnement ammoniaque et morpholine (Centrale de Fessenheim). Mesures d'hydrogène dans les circuits eau-vapeur (moyennes journalières).

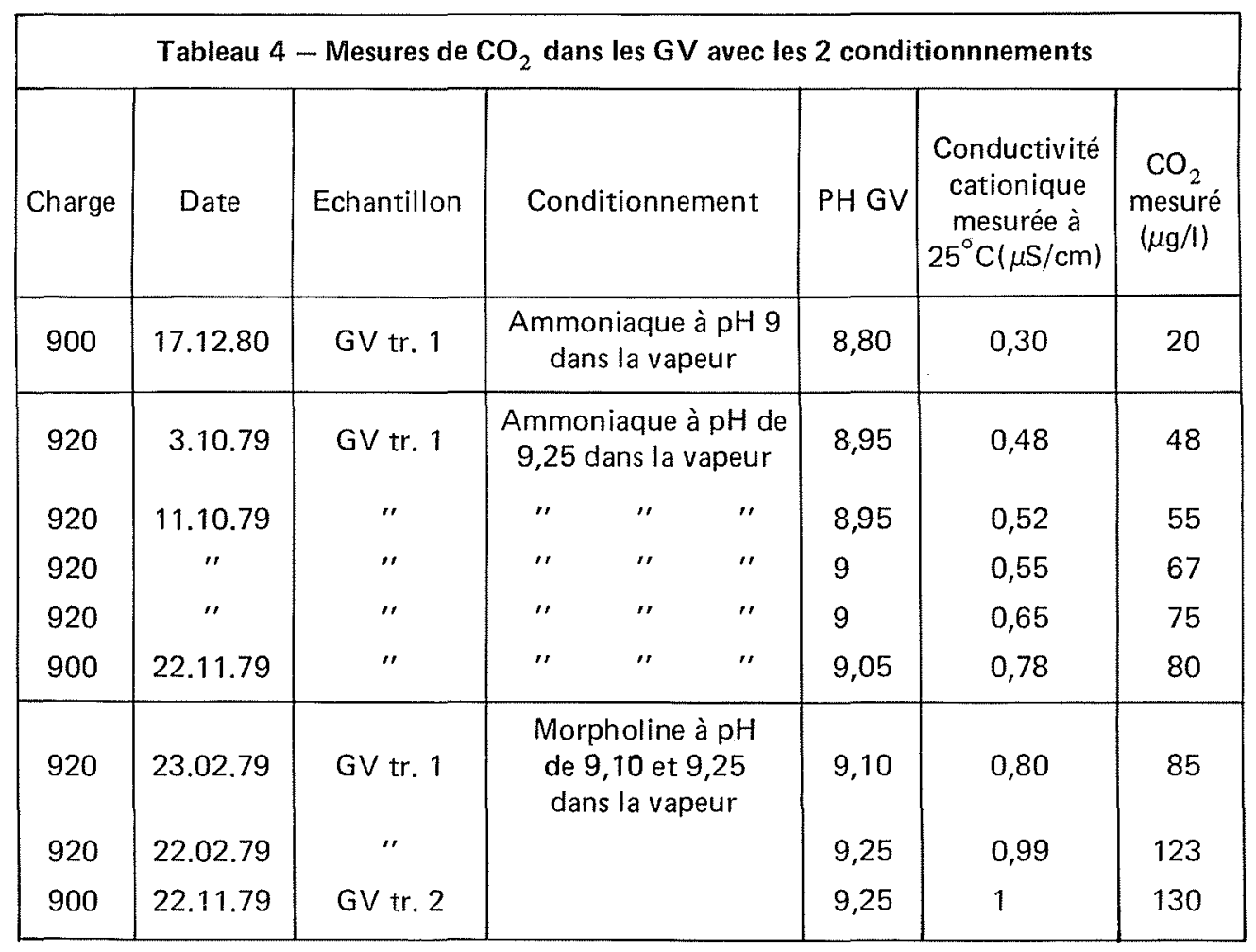


pour la phase eau + morpholine que pour la phase eau + ammoniaque (cette dernière ayant un $\mathrm{pH}$ plus faible).

Ceci peut s'appliquer en tout point du circuit secondaire où intervient une séparation des phases eau et vapeur : GV, sécheurs surchauffeurs, condenseur.

Influence du $\mathrm{CO}_{2}$ sur les mesures de conductivité cationique des générateurs de vapeur avec les deux conditionnements

On constate à la centrale de Fessenheim, en marche normale, sans entrée d'eau brute, des $\lambda^{+}$GV plus élevées en conditionnement morpholine qu'en conditionnement ammoniaque (de l'ordre de 0,3 à $0,4 \mu \mathrm{S} / \mathrm{cm}$ à $25^{\circ} \mathrm{C}$ ).

Cette différence n'a pu être expliquée par la présence d'impuretés de la morpholine ou des produits résultant de sa décomposition thermique. On constate en effet, qu'à la température de $300^{\circ} \mathrm{C}$ maximale des circuits, une telle décomposition ne semble pas se produire. Ceci a pu être mis en évidence, dès le début des essais de conditionnement morpholine sans hydrazine en tranche 1, par l'absence d'ammoniaque, produit de la décomposition thermique de la morpholine, facilement identifiable.

Nos essais ont permis de constater qu'une augmentation de l'ordre de 0,2 à $0,3 \mu \mathrm{S} / \mathrm{cm}$ de la conductivité cationique dans les $\mathrm{GV}$ :

- d'une part entre un conditionnement ammoniaque à $\mathrm{pH} 9$ dans la vapeur et le même conditionnement à pH 9,25 ;

- d'autre part entre un conditionnement ammoniaque à $\mathrm{pH} 9,25$ dans la vapeur et un conditionnement morpholine à même $\mathrm{pH}$,

correspond à une augmentation de la teneur en $\mathrm{CO}_{2}$ due à la seule élévation du $\mathrm{pH}$ dans les $\mathrm{GV}$ (voir tableau 4).

Cependent, la teneur en $\mathrm{CO}_{2}$ dans les $\mathrm{GV}$ ne permet pas de rendre compte de toute l'augmentation des conductivités cationiques GV. On peut donc faire deux hypothèses :

1) Il existe une impureté marquant la conductivité cationique GV et qui croîtrait, quel que soit le conditionnement, en fonction du pH des $G V$, ce qui est peu vraisemblable.

2) Le $\mathrm{CO}_{2}$ est dosé systématiquement par défaut.

Etant donné les difficultés de cet ordre déjà rencontrées, cette $2^{\mathrm{e}}$ hypothèse apparaît comme étant la plus plausible.

\section{Conclusions}

Le conditionnement morpholine apparaît comme le meilleur conditionnement susceptible de protéger :

- les tuyauteries de liaison HP, les sécheurs-surchauffeurs en acier au carbone et certains organes de la turbine HP, des phénomènes d'érosion-corrosion en vapeur humide; - les refroidisseurs d'air en laiton des condenseurs de la corrosion ammoniacale.

$\mathrm{Au}$ niveau des $\mathrm{GV}$, il permet, en considérant les matériaux des équipements précédents, d'introduire moins de matières en suspension. De plus, en cas de présence importante de $\mathrm{CO}_{2}$ en démarrage ou de micro entrées d'eau brute à sels hydrolysables acides, il permet de tamponner dans une certaine limite le $\mathrm{pH}$ des GV. Cet effet tampon risque toutefois d'être moins marqué dans les zones de concentrations locales qui sont précisément celles qui sont affectées par le denting.

Le conditionnement morpholine présente en outre comme avantage par rapport au conditionnement ammoniaque le maintien d'un $\mathrm{pH}$ stable et identique dans les phases eau et vapeur.

Ce conditionnement est d'ailleurs utilisé sans difficultés majeures d'exploitation dans les centrales de Chooz et Tihange.

Par contre il présente l'inconvénient d'augmenter sensiblement les conductivités cationiques de l'eau des GV ce qui entraine :

- une augmentation du capital conductivités cumulées $\left(\mu \mathrm{S} . \mathrm{cm}^{-1} \cdot \mathrm{j} / \mathrm{an}\right)$

- une diminution de la sensibilité de détection des entrées d'eau brute au condenseur.

Un dispositif permettant de mesurer directement la conductivité cationique $\mathrm{GV}$ due aux sels d'acide fort sans l'influence du $\mathrm{CO}_{2}$ est actuellement en essai industriel à la centrale de Fessenheim. Il devrait permettre s'il s'avère exploitable, de supprimer l'inconvénient des conductivités dues aux teneurs en $\mathrm{CO}_{2}$, plus importantes en conditionnement morpholine qu'en conditionnement ammoniaque.

La plupart des matériaux susceptibles de détérioration chimique étant dès à présent remplacés par des matériaux plus nobles (acier inox, titane), ce conditionnement morpholine, dans l'état actuel de nos connaissances, n'est donc plus à recommander que pour les unités sur lesquelles ces changements ne sont pas réalisés.

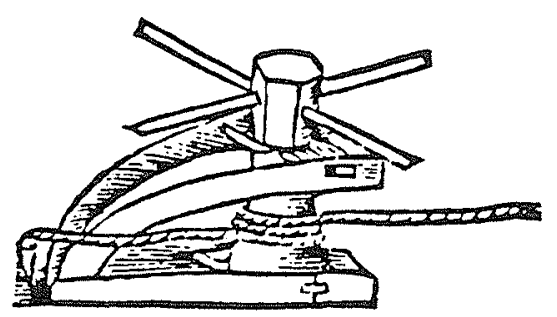




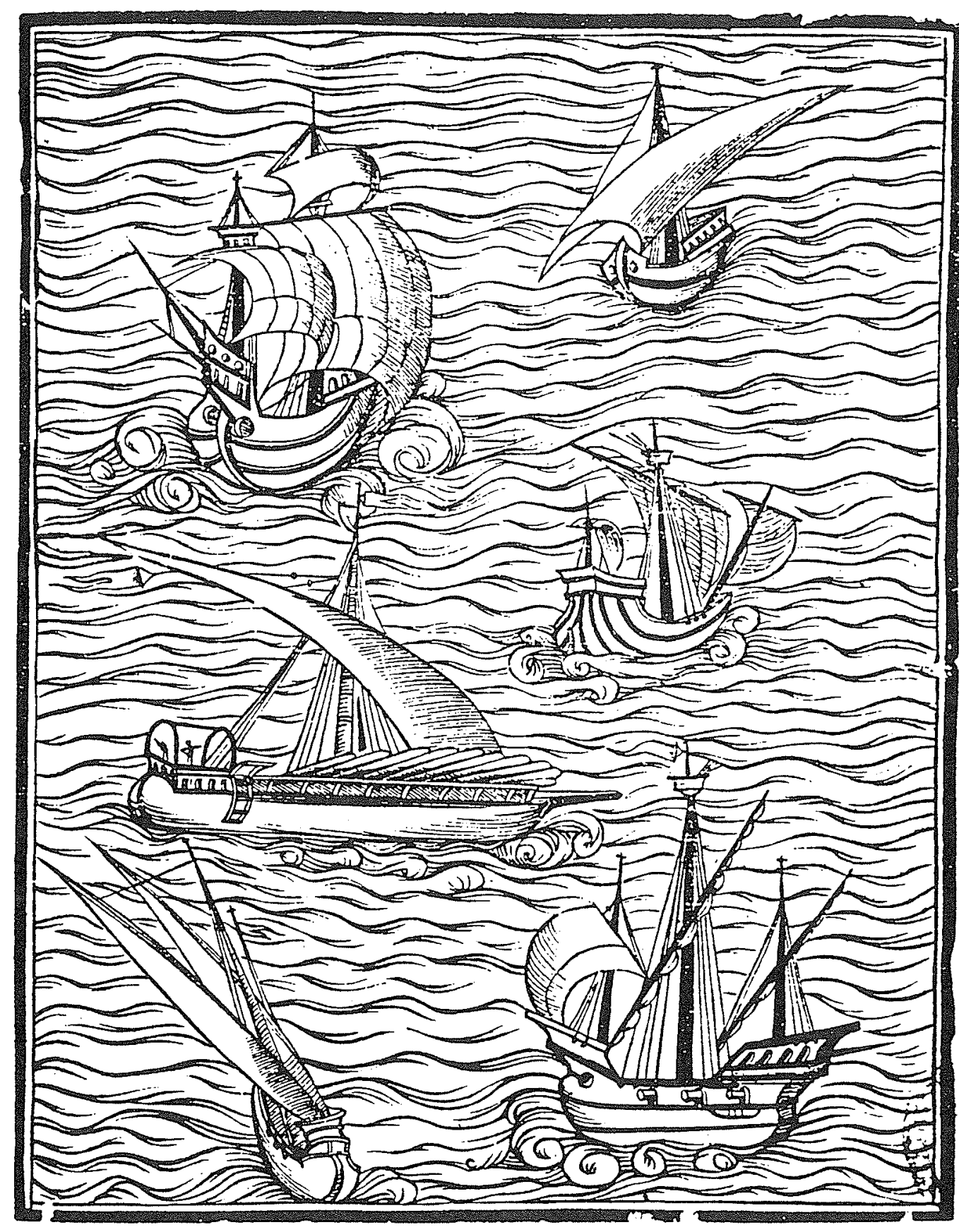

